\title{
Dynamic reorganization of the frontal parietal network during cognitive control and episodic memory
}

\author{
Kimberly L. Ray ${ }^{1}$ • J. Daniel Ragland ${ }^{1} \cdot$ Angus W. MacDonald $^{2} \cdot$ James M. Gold $^{3} \cdot$ Steven M. Silverstein $^{4}$. \\ Deanna M. Barch ${ }^{5}$ - Cameron S. Carter ${ }^{1,6,7}$
}

Published online: 6 December 2019

(C) The Psychonomic Society, Inc. 2019

\begin{abstract}
Higher cognitive functioning is supported by adaptive reconfiguration of large-scale functional brain networks. Cognitive control (CC), which plays a vital role in flexibly guiding cognition and behavior in accordance with our goals, supports a range of executive functions via distributed brain networks. These networks process information dynamically and can be represented as functional connectivity changes between network elements. Using graph theory, we explored context-dependent network reorganization in 56 healthy adults performing fMRI tasks from two cognitive domains that varied in CC and episodic-memory demands. We examined whole-brain modular structure during the DPX task, which engages proactive CC in the frontal-parietal cognitive-control network (FPN), and the RiSE task, which manipulates CC demands at encoding and retrieval during episodicmemory processing, and engages FPN, the medial-temporal lobe and other memory-related networks in a context dependent manner. Analyses revealed different levels of network integration and segregation. Modularity analyses revealed greater brainwide integration across tasks in high CC conditions compared to low CC conditions. Greater network reorganization occurred in the RiSE memory task, which is thought to require coordination across multiple brain networks, than in the DPX cognitivecontrol task. Finally, FPN, ventral attention, and visual systems showed within network connectivity effects of cognitive control; however, these cognitive systems displayed varying levels of network reorganization. These findings provide insight into how brain networks reorganize to support differing task contexts, suggesting that the FPN flexibly segregates during focused proactive control and integrates to support control in other domains such as episodic memory.
\end{abstract}

Keywords Cognitive control $\cdot$ Episodic memory $\cdot$ Functional connectivity $\cdot$ Neural network

Electronic supplementary material The online version of this article (https://doi.org/10.3758/s13415-019-00753-9) contains supplementary material, which is available to authorized users.

Cameron S. Carter

cscarter@ucdavis.edu

1 Imaging Research Center, University of California at Davis, Sacramento, CA, USA

2 Department of Psychology, University of Minnesota, Minneapolis, MN, USA

3 Maryland Psychiatric Research Center, University of Maryland School of Medicine, Baltimore, MD, USA

4 Department of Psychiatry, Rutgers - Robert Wood Johnson Medical School, Piscataway, NJ, USA

5 Department of Psychiatry, Washington University in St. Louis, St Louis, MO, USA

6 Department of Psychology, University of California at Davis, Davis, CA, USA

7 Department of Psychiatry, University of California at Davis, $4701 \mathrm{X}$ Street, Sacramento, CA 95817, USA

\section{Introduction}

Cognitive control (CC) plays a vital role in flexibly guiding cognition and behavior in accordance with our goals, and it is thought that this ability serves as an important element in healthy brain function (Veen \& Carter, 2006). This mechanism is not limited to a particular cognitive domain (Banich, 1997), CC supports a range of cognitive functions, including working memory, episodic memory (Ragland et al., 2009), inhibitory processing (Banich et al., 2000), and goal maintenance (Henderson et al., 2012). These aspects of executive functions are supported by distributed brain networks that represent and process information in a dynamic manner via functional connectivity (FC) between network elements (Cole \& Schneider, 2007; Cole et al., 2013).

The prefrontal cortex (PFC) plays a central role in cognitive control (Badre, 2008; MacDonald, 2000; Niendam et al., 2012). Evidence supports an anterior-posterior gradient of function within the PFC. While the rostrolateral PFC is 
associated with relational reasoning, functional magnetic resonance imaging (fMRI) studies suggest that interactions between dorsal and ventral lateral prefrontal regions and posterior brain regions including the lateral parietal lobe and the medial temporal lobe (MTL) support the retrieval of relationally encoded information and associative recognition during episodic memory (Murray \& Ranganath, 2007; Ragland et al., 2012, 2015). More broadly, the evidence for such segregated brain activity during the processing of goal maintenance is consistent with recent studies suggesting that trial-by-trial cognitive control engages a large-scale functional brain network encompassing frontal and parietal cortices (Henderson et al., 2012; Lopez-Garcia et al., 2015).

Advances in applying graph analysis to fMRI data have provided means to mathematically describe and quantify cognition-related patterns of function connectivity. Initially restricted to rest, developments in graph theory (GT) methodology applied to task fMRI have furthered our understanding of how cognitive control is supported through flexible, context-dependent integration and segregation of functional brain networks in a dynamic manner (Braun et al., 2015; Cocchi et al., 2013a; Cocchi et al., 2013b; Fornito et al., 2012; Hearne et al., 2017). These studies commonly focus on network organization supporting performance of a single cognitive task within a single brain network or defined set of networks. More recently, studies have demonstrated that modular properties of brain networks shift in response to differing cognitive demands (Cohen \& D'Esposito, 2016; Geib et al., 2017; Westphal et al., 2017). The current fMRI study examines whole brain network organization involved in cognitive control during two tasks that involve multiple cognitive domains. We examine network reorganization specifically within the framework of changes in functional connectivity where we investigate two different mechanisms of reorganization: (1) enhanced network connectivity in the frontal parietal network (FPN) using the participation coefficient and (2) enhanced between network connectivity, using modularity, where cognitive systems integrate to create new networks.

We use modularity, a GT method that measures the decomposability of a graph into modules or communities, to provide insight into context-dependent network reorganization in healthy adults (HC) performing tasks with varying demands on cognitive control. Modularity is a general hallmark of complex biological systems. Modular organization of brain networks shapes how information is distributed and processed where regions that are functionally close and tend to share information are considered members of the same cluster or module (Sporns \& Betzel, 2016). A module containing nodes from a variety of cognitive systems likely indicates functional integration amongst cognitive brain networks, whereas a module composed of only nodes from a single system may likely represent network segregation.
Reliability of the brain's functional architecture at rest is well supported; however, recent GT analyses have revealed an adaptive reconfiguration of large-scale brain networks that support higher cognitive functions (Bassett et al., 2011; Braun et al., 2015; Cole et al., 2014; Hearne et al., 2017). Studies probing the correspondence of network organization during rest and task using residualized and non-residualized fMRI data have found that network modifications during task were independently associated with regional activation and changes in functional connectivity (Gratton, Laumann, Gordon, Adeyemo, \& Petersen, 2016). Ultimately this suggests that meaningful, context-dependent network reconfigurations occur against a backdrop of stable, large-scale networks that support diverse cognitive functions (Cohen \& D'Esposito, 2016; Cole et al., 2014; Crossley et al., 2013; Hearne et al., 2017).

The current study examines cognitive-control processing in data from 56 healthy adults performing fMRI tasks from two distinct cognitive domains that varied in demands for cognitive control, the RiSE episodic memory task and the Dot Pattern Expectancy (DPX) goal maintenance task. Adapting a beta series correlation technique (Mumford et al., 2012) to examine brain-wide integration and segregation of cognitive systems during the RiSE and DPX, we leverage opposing network topology to quantitatively assess the dynamic network reorganization (i.e., variation in community structure corresponding to diverse cognitive-control demands) involved in each of these cognitive tasks. Prior work from this sample used the Network Based Statistic (Zalesky et al., 2010) to identify increases in functional connections within the FPN associated with cognitive-control demand (Ray et al., 2017). However, it is not clear how within-network FC changes correspond to brain-wide network organization. Here, we investigate context-dependent network reorganization via changes in integration and segregation properties associated with modular organization across the RiSE and DPX tasks. We predict that increased demands for cognitive-control processing will result in greater brain-wide integration of cognitive systems measured by decreased modularity. We measure changes in modular partitions associated with increased $\mathrm{CC}$ using mutual information. We hypothesize that the FPN will integrate with other higher-cognitive networks in order to support efficient cognitive processing/task completion, which we quantify by means of increased participation coefficient of modular partitions.

\section{Materials and methods}

\section{Subjects}

Study participants were recruited as part of the CNTRACS Consortium (http://cntracs.ucdavis.edu), which included five different research sites: University of California - Davis, 
Maryland Psychiatric Research Center at the University of Maryland, Rutgers University - Robert Wood Johnson Medical School, University of Minnesota - Twin Cities, and Washington University. Recruitment and informed consent procedures for each site were approved by their Institutional Review Boards. Complete details regarding CNTRACS recruitment and enrollment can be found in Ragland et al. (2015).

Data were obtained on 60 healthy adults (HC). Participants were excluded if they exhibited excess movement (i.e., > $0.37 \mathrm{~mm}$ mean frame-to-frame movement), below-chance performance, or image acquisition errors. This left final samples of $56 \mathrm{HC}$ ( $34.0 \pm 11.4$ years) for the RiSE task, and $52 \mathrm{HC}$ (34.1 \pm 10.4 years) for the DPX task (Table 1$)$.

While fMRI and behavioral data from these subjects have been used in previous publications, results from the current study are unique and do not include previously published findings.

\section{Data acquisition}

Relational and Item-Specific Encoding (RiSE) task The design was identical to that of the original RiSE studies (Ragland et al., 2012, 2015), with the following exceptions: stimuli were presented in pairs during both encoding conditions (see below), and the item-recognition task did not include confidence ratings. Participants completed four encoding and four recognition fMRI runs. During encoding (Fig. 1A), participants alternated between three item-specific blocks ("Is either object living?"; nine low cognitive-control trials each) and three relational blocks ("Can one object fit inside the other?"; nine high cognitive-control trials each) in a "jittered" eventrelated design. During item recognition (Fig. 1B), participants made a two-button response to indicate whether objects were previously studied (old) or never studied (new). During item recognition, 54 individual objects from each encoding condition (54 item-specific, 54 relational) were randomly presented with 54 new items. The Rise task is considered a rapidpresentation event-related fMRI paradigm, therefore

Table 1 Participant demographics

\begin{tabular}{ll}
\hline & $\begin{array}{l}\text { HCs (n=56) } \\
\text { Mean (SD) }\end{array}$ \\
\hline Age, y & $33.98(11.40)$ \\
WTAR & $37.89(10.2)$ \\
Education, y & \\
Participant & $14.84(1.87)$ \\
Parent & $14.9(3.9)$ \\
Male sex, no. (\%) & $40(71 \%)$ \\
Right-handed, no. $(\%)$ & $52(93 \%)$ \\
\hline
\end{tabular}

HCs healthy controls, WTAR Wechsler Test of Adult Reading
OPTSEQ (available at https://surfer.nmr.mgh.harvard.edu/ optseq/) was used to optimize the efficiency of trial presentation timing and randomization across each block. Because our interest was in engagement of $\mathrm{CC}$ during encoding processes rather than accuracy of frequently equivocal responses (e.g., Is an apple that is not on the tree living?), fMRI analysis included trials in which participants correctly responded during the recognition condition and their corresponding encoding trials. As reported in Ragland et al. (2015), mean accuracy for healthy adults was $72.0 \%$ and 86 . $1 \%$ for item and relational recognition trials, respectively. See Ragland et al. (2015) for more information regarding the RiSE task.

Previous RiSE fMRI studies contrasting relational (high cognitive control) against item-specific (low cognitive control) in the same sample have identified robust activation increases in the bilateral DLPFC, VLPFC, parietal, and occipital cortices (Ragland et al., 2015). Furthermore, functional connectivity analyses examined in this sample have demonstrated network-specific engagement of the FPN during the RiSE task (Ray et al., 2017).

Dot Pattern Expectancy (DPX) task The DPX task consisted of a sequence of cue-probe stimuli where participants made one response when a target cue-probe pair was presented and another response for all other stimuli (Fig. 2). Cues indicated the need for high (B Cues) or low (A Cues) levels of cognitive control. Four types of trials were presented across four blocks: $\mathrm{AX}, \mathrm{AY}, \mathrm{BX}$, and BY. AX trials are "target trials," where a valid cue is followed by a valid probe. The three other trial types are "Non-target trials" in which either a valid cue is followed by an invalid probe ("AY" trials) or an invalid cue is followed by either a valid or invalid probe ("BX" or "BY" probes, respectively). Each block of the DPX task consisted of 40 trials: 24 AX, six AY, six BX, and four BY. The nature of the cue (valid or invalid) provides the "context" for responding on a given trial. The majority of trials are "target trials" (AX trials). This feature is intended to encourage participants to "expect" a valid probe to follow a valid cue. A consequence of this manipulation is that participants develop a prepotency to respond with "target" responses on trials for which valid cues are presented, regardless of whether the trials were of the target (AX) or non-target (AY) type. The DPX task is a rapid-presentation event-related fMRI paradigm, therefore OPTSEQ (available at https://surfer.nmr.mgh.harvard.edu/ optseq/) was used to optimize the efficiency of trial presentation timing and randomization across each block. Correct responses from four runs of the DPX task were used for analysis. See Poppe et al. (2016) for more detail regarding the AX-DPX.

Previous fMRI whole-brain analyses from our group using a different healthy sample have shown that contrasting B-cues relative to A-cues in the DPX task elicits widespread 
a Encoding Item Encoding
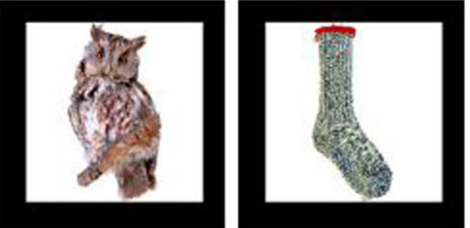

Living?

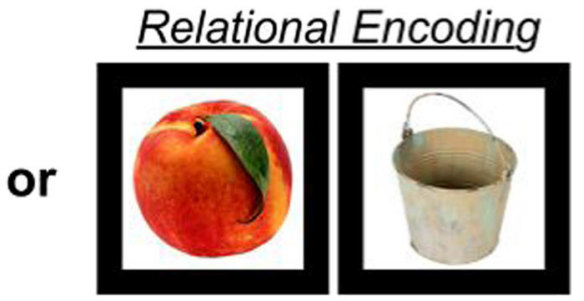

Inside?

\section{b Item Recognition}

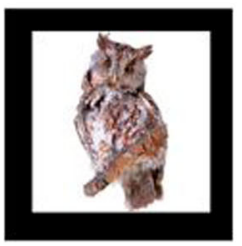

Old?

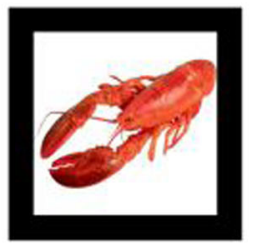

Old?

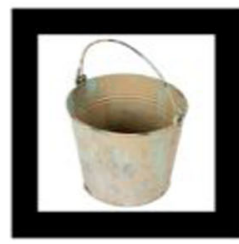

Old?
Fig. 1 Illustration of the RiSE task. (A) Item specific (left) and relational (right) object pairs presented while subjects made either an item-specific encoding response or a relational encoding response. (B) During item recognition, objects from item and relational encoding conditions were randomly presented with new items, and participants indicated whether each item was old (i.e., previously studied)

\section{Preprocessing}

DLPFC, bilateral fusiform gyri, and right inferior parietal gyrus (Lopez-Garcia et al., 2015). These activation findings have been replicated in the current sample (Poppe et al., 2016) and subsequent functional connectivity analyses have also demonstrated network-specific engagement of the FPN during the DPX task (Ray et al., 2017).
Images were acquired in a single $3 \mathrm{~T}$ MRI session using a consistent protocol across sites. Functional images were acquired using gradient-echo BOLD echo-planar imaging $(\mathrm{TR}=$ $2,000 \mathrm{~ms}, \mathrm{TE}=30 \mathrm{~ms}, 77^{\circ}$ flip angle, $\mathrm{FOV}=220 \mathrm{~mm}^{2}, 3.43 \times$ $3.43 \times 4 \mathrm{~mm}$ voxels, 32 axial slices parallel with the anterior/

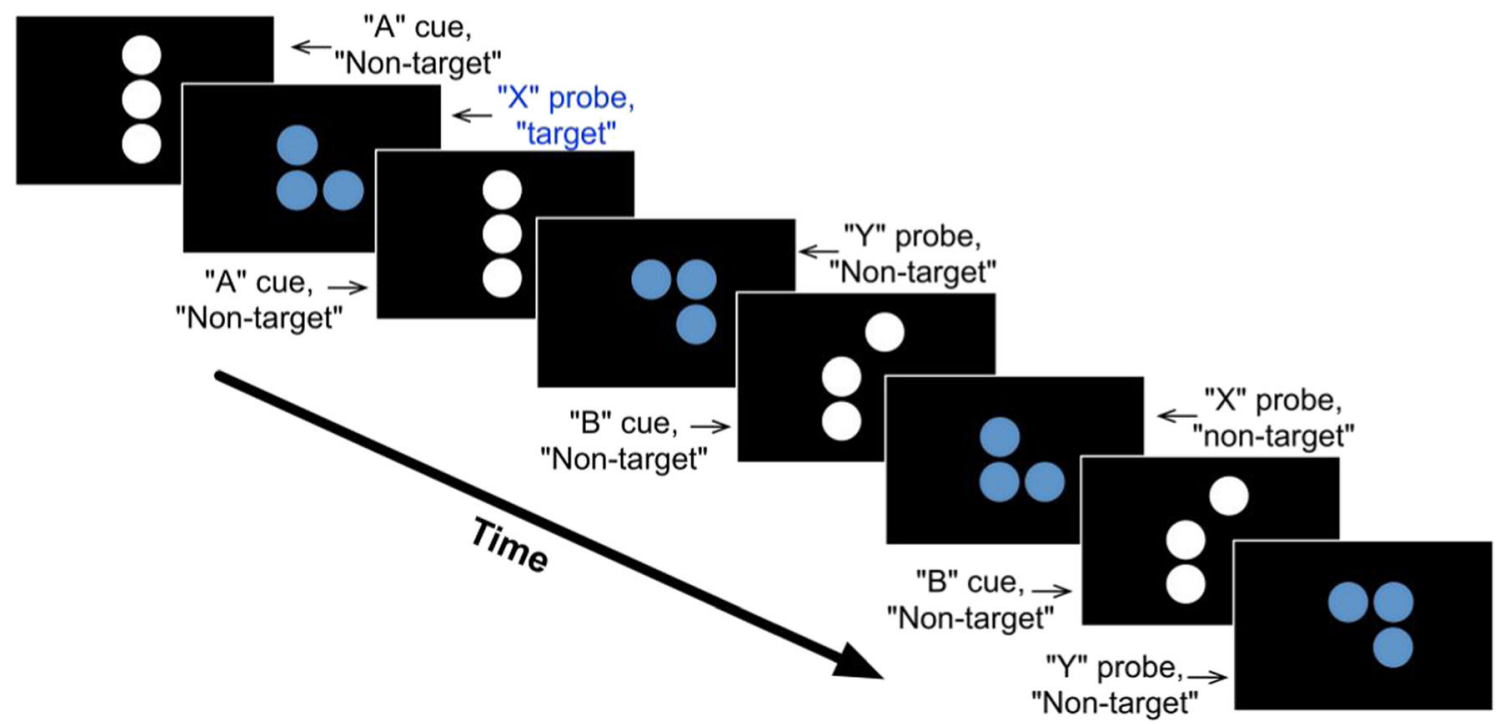

Fig. 2 Illustration of the Dot Pattern Expectancy Task. Shown is an example sequence of cue-probe stimuli and the type of response (target or non-target) a participant was required to make after each stimulus. The nomenclature for stimuli and trial types was adopted from the expectancy letter AX task. The valid cue pattern is referred to as " $A$ " and the valid probe pattern is referred to as "X." Non-"A" cue patterns are referred to as
"B"-type cues, and non-"X" probe patterns are referred to as "Y"--type probes. A target response is required for " $\mathrm{X}$ " when it follows " $\mathrm{A}$," nontarget responses are made for all other stimuli. The first pair of stimuli in the sequence represents an AX trial. The third and fourth stimuli together represent an AY type of trial, the fifth and sixth stimuli together complete a BX trial, and the seventh and eighth stimuli make up a BY type of trial 
posterior commissure). For more information see Henderson et al. (2012).

Pre-processing was carried out using the FMRI Expert Analysis Tool (FEAT) in the FMRIB Software Library (FSL version 4.1; www.fmrib.ox.ac.uk/fsl) using standard procedures, including field-map correction, spatial normalization, and nonlinear registration to MNI152. Field maps to correct fMRI data for geometric distortion caused by magnetic field inhomogeneity and a T1-weighted anatomical image (1$\mathrm{mm}$ isotropic voxels) were also acquired.

\section{Data processing (beta-series regression)}

Subject-wise beta-series regression analysis was performed on RiSE and DPX fMRI data in order to capture trial-specific BOLD effects for each condition (Turner et al., 2012). Using the least-squares-separate (LS-S) method to measure eventrelated functional connectivity, individual trials were modeled with a new GLM in SPM8 with two predicted BOLD time courses - one that reflects the expected BOLD response to the current event and another for the BOLD responses to all events except the current event. All events were modeled, but only cue events for DPX correct trials and correct RiSE trials were included in the analysis because these represented trial periods in which cognitive-control demands were maximized.

Separate regressors modeling each event were defined in a general linear model to yield unique condition-wise beta values for every voxel. Each beta value reflected the magnitude of the hemodynamic response evoked by each event. Beta images were sorted by condition and concatenated across runs yielding a $4 \mathrm{D}$ dataset (space $\times \mathrm{n}$ trials), or beta-series, for each of the six conditions: RiSE Item Encoding, RiSE Relation Encoding, RiSE Item Recognition, RiSE Relation Recognition, DPX A Cue, and DPX B Cue.

Additional motion-correction steps were taken analogous to data-scrubbing procedures often performed in resting-state functional connectivity analyses (Power et al., 2014). Betaimages containing frame-wise displacement (FD) values greater than $0.5 \mathrm{~mm}$ motion were excluded from the betaseries. If more than $10 \%$ of TRs within a block contained frame-wise displacement $>0.5 \mathrm{~mm}$, the entire block was excluded from analysis. This FD threshold led to the exclusion of five blocks of the RiSE task, and one block in the DPX task.

Next, each participant's brain data were parcellated into discrete regions of interest representing nodes obtained from the Power atlas (Power et al., 2011). Twenty-one Power nodes were eliminated due to low signal, and two bilateral MTL nodes were added (MNI coords: -30,-12,-22; 32,-14,-22) resulting in 245 nodes across the whole brain. Beta-series pairwise correlations for all 245 nodes were extracted and ztransformed resulting with a $245 \times 245$ connectivity matrix. A final group FC graph for each condition was established by summing the $5 \%$ thresholded connection matrices across all subjects, then applying a subsequent $5 \%$ threshold on the summed group connection matrix to identify connections that are consistently strongest across participants, followed by binarization of the summed group connection matrix.

\section{Graph analysis}

Recent advances in the application of graph theoretical analysis to fMRI data allow us to leverage information contained within the BOLD signal to test hypotheses regarding the functional architecture of the human brain (Bullmore \& Bassett, 2011). One well-known investigation of community structure in functional brain networks (Power et al., 2011) extracted communities from restingstate fMRI data and, using a map of task-based activations across a range of tasks, mapped these communities to wellstudied cognitive systems. The current study examines segregation and integration of brain networks via modular organization during each condition in the RiSE and DPX tasks separately and leverages opposing network topology to highlight the dynamic reorganizations that support these different cognitive-control tasks in healthy adults. That is, we used graph theoretical measures (i.e., modularity) to model and quantify context-dependent changes in functional brain organization during three fast event-related fMRI paradigms: The RiSE encoding, RiSE recognition, and DPX tasks.

Modularity Modularity (Q) and modular partitions were extracted for each subject using the community Louvain algorithm provided by the Brain Connectivity toolbox (Rubinov \& Sporns, 2010). A Louvain module partition yields a set of non-overlapping communities, where each node is assigned to one and only one module. Modularity $(\mathrm{Q})$ ranges from zero to one and quantifies the goodness of modular partitions, where good modular partitions have high modularity values and an unexpectedly high proportion of connections within modules, and an unexpectedly low proportion of connections between modules (Newman, 2004). A high proportion of connections between modules (i.e., low modularity) suggests a greater level of brain-wide community integration amongst cognitive systems, whereas a low proportion of connections between modules (i.e., high modularity) may suggest a segregation of communities. Nevertheless, the Louvain modularity algorithm uses a randomized heuristic approach and consequently results across runs slightly vary. We therefore applied this algorithm 1,000 times for each task condition at proportional thresholds of 0.05 through 0.60 (to avoid negative connections in FC matrices) at increments of 0.05 , and selected the partition with the highest modularity score, Q (Meunier, 2009; Rubinov \& Sporns, 2011). Modularity analyses were 
run on the group connection matrix and on individual subjectconnection matrices for each task.

Comparison of task-based $\mathbf{Q}$ to null model Null models are important adjuncts of descriptive graph analysis as they allow discriminating which graph attributes are due to chance and which exceed the expected values given by the null model (Sporns, 2018). To establish a null model for each condition examined, we assessed the modularity of random graphs derived from each task condition. Random graphs for each participant and for the group mean were created using functions provided in the Brain Connectivity Toolbox that randomize connections in their corresponding task-based network (randmio_und.m, 500 iterations), while preserving the degree distribution (Rubinov \& Sporns, 2011). Modularity (Q) of task-based graphs were compared to their respective null model across a range of thresholds (i.e., $0.05-0.60$ proportional thresholds at intervals of 0.05 ) using a repeated-measures ANOVA.

Comparison of task-based $\mathbf{Q}$ to Power $\mathbf{Q}$ An advantage of using the Power atlas is that it also provides an a priori partition of subgraphs that replicate across cohorts and correspond anatomically with many functional systems consistently observed in the neuroimaging literature (Power et al., 2011). As a follow-up to task-based modularity analyses of the RiSE and DPX paradigms, we were interested in comparing the task-based Louvain-Modular partitions to the Power atlas resting-state subgraph partition. To do so, modularity $(\mathrm{Q})$ of the Power resting-state subgraph partition was calculated for each subject using an abridged version of modularity scripts provided in the BCT that determines $\mathrm{Q}$ based on the input Power partition rather than an optimized partition. Modularity of the Power partition for each subject was then compared to the modularity of their corresponding task-based partitions using a repeated-measures ANOVA.

Within-network connectivity Changes in functional network organization are a byproduct of functional connectivity changes. To better understand which cognitive systems likely contribute to changes in network organization, the average connectivity (mean Fisher $\mathrm{z}$ value) across all ROI-ROI connections within each of the Power networks was computed. We used a repeated-measures three-way ANOVA to examine whether main effects of task, cognitive control, or Power network were present. This was followed by separate two-way repeated-measures ANOVAS to test for main effect cognitive control within each Power network.

Quantifying network reorganization using mutual information theory We utilized popular information-theoretic measures of distance in partition space, mutual information and variation of information (Meilă, 2007; Rubinov \& Sporns, 2011), to provide a measure of the amount of network reorganization between high and low controlcondition partitions in the RiSE and DPX tasks. Using functions provided in the Brain Connectivity Toolbox (partition_distance.m; Rubinov \& Sporns, 2011), mutual information between two partitions $M$ and $M^{\prime}$ was calculated as:

$I\left(M, M^{\prime}\right)=\sum_{u \in M} \sum_{u^{\prime} \in M^{\prime}} P\left(u, u^{\prime}\right) \log \frac{P\left(u, u^{\prime}\right)}{P(u) P\left(u^{\prime}\right)}$

where $P\left(u, u^{\prime}\right)=\frac{n_{u u^{\prime}}}{n}$ and $n_{u u^{\prime}}$ is the number of nodes that are simultaneously in module $u$ of partition $M$, and in module $u$ ' of partition $M^{\prime}$. Variation of information is defined as:

$V I=\frac{1}{\log n}\left[H(M)+H\left(M^{\prime}\right)-2 I\left(M, M^{\prime}\right)\right]$,

where the factor $\frac{1}{\log n}$ rescales the variation of information to the range of $[0,1]$, such that $\mathrm{VI}=0$ corresponds to equal partitions, and VI $=1$ corresponds to maximally different partitions (Rubinov \& Sporns, 2011). Partition vectors $M$ and $M^{\prime}$ contained the full set of 245 nodes, thus mutual information and variation of information values reflect network reorganization across the whole brain unless otherwise noted.

In the context of the current study, mutual information indicates the degree to which nodes are similarly assigned to modules during high and low cognitive-control conditions. A mutual information (I) value of one would indicate two identical partitions are being compared and no change in module organization between high and low cognitivecontrol conditions. A mutual information index of zero would indicate maximally different partitions and thus a large change in module organizations between the two conditions compared.

Participation Coefficient We utilized the participation coefficient (PC) to examine the integrative role of the FPN with respect to other cognitive networks. The participation coefficient measures the proportion of connections a node has within its own module versus other modules in the network (Guimerà \& Nunes Amaral, 2005; Rubinov \& Sporns, 2010; Sporns et al., 2007). Thus, nodes with high PC are more strongly connected to nodes associated with other modules, thereby facilitating greater integration of information across modules; in contrast, nodes with lower PC are predominately connected to nodes within its assigned module. The PC of FPN nodes in the Power atlas were 
extracted from the 5\% thresholded functional connectivity matrices of each task condition for each individual participant. PCs were averaged across all nodes within the FPN, and a repeated-measures $3 \times 2$ ANOVA was performed to test the effect of cognitive control across the RiSE encoding, RiSE recognition, and DPX tasks.

\section{Results}

\section{Comparison of task-based $\mathbf{Q}$ to null model}

Subject-wise functional partitions thresholded to the strongest $5 \%$ of connections were significantly more modular $(\mathrm{Q})$ than their respective null models for each task, $F(1,51)=2497, p<$ 0.001 (Fig. 3). This significant difference was present across a range of thresholds.

\section{Comparison of task-based Q to Power Q}

Compared to modularity (Q) of the a priori Power partition, we found that task-based functional partitions provided a significantly greater modularity score than the restingstate subgraph partition $(F(1,51)=9710.173, p<0.001$; $5 \%$ threshold). A greater Q-score indicates that newly established task modules yield a better model of network organization during the RiSE and DPX paradigms than the resting-state Power subgraph partition, and that a reasonable amount of network reconfiguration occurs between rest and task (Hearne et al., 2017). Moreover, Power modularity scores $(\mathrm{Q})$ were, on average, 0.013 higher than their null models across tasks $(F(1,51)=6.541, p=0.014$; $5 \%$ threshold).

\section{Task-based modularity}

Once the non-randomness of task-based modular partitions was established across participants, we performed a $3 \times 2$ ANOVA to examine the effects of task and cognitive control. In doing so, we observed an overall effect of task $(F(2,50)=9.615, p<0.001)$, indicating that the RiSE and DPX tasks exhibit different levels of network integration and segregation (Fig. 3B). A cognitive control effect was also present, where low cognitive-control conditions exhibited greater modularity relative to high control conditions across RiSE and DPX conditions $(F(1,51)=5.673$, $p<0.021)$. Furthermore, a task-by-control interaction effect was present $(F(1,51)=3.179, p<0.046)$, indicating that while low cognitive-control conditions were greater than high cognitive-control conditions in each of the tasks examined, that the extent of this modularity effect varied across RiSE encoding, RiSE recognition, and DPX tasks.

\section{Within-network connectivity}

Considering that changes in network organization (e.g., modular composition, participation coefficient) are a result of underlying changes in functional connectivity, we examined within-network connectivity of each cognitive network in the Power atlas to better understand which networks likely

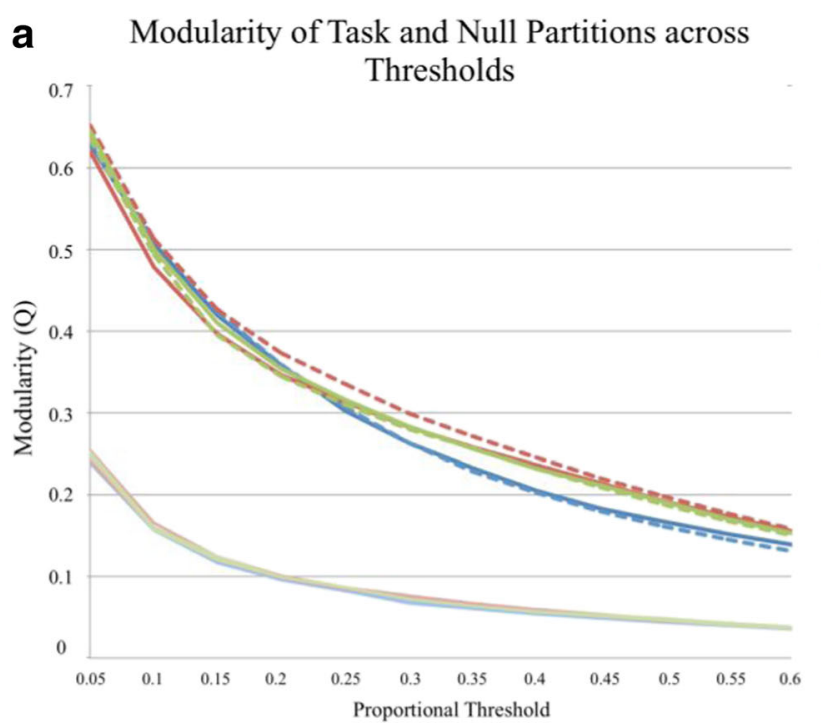

Fig. 3 (A) Louvain modularity (Q) of group mean task-based Louvain partitions compared to their respective individual null-model. Task-based partitions were significantly more modular than their null model across all thresholds tested $(p<0.001)$. (B) Modularity of each individual's taskbased partitions at a 0.05 proportional threshold compared to their null-

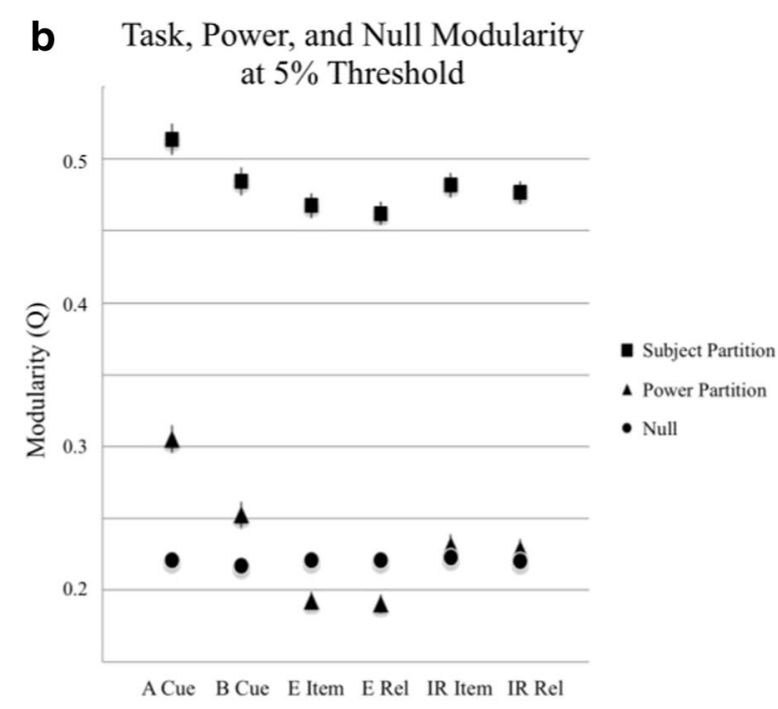

model and the Power subgraph partition. A Cue and B Cue refer to the two DPX conditions. E Item and E Rel refer to the RiSE item and relational encoding conditions, respectively. IR Item and IR Rel refer to the RiSE item and relational Recognition conditions, respectively 
contribute to reorganization during cognitive-control processing. First, a three-way repeated-measures ANOVA was performed to test for main effects of task, cognitive control, and Power network. While effects of cognitive control $(F(1,2,13)=$ $1.269, n s)$ and task $(F(1,2,13)=1.765, n s)$ were not significant, there was a main effect of Power Network $(F(1,2,13)=76.134$, $p<0.001$ ), indicating that cognitive systems displayed different strengths of connectivity during the cognitive control. Separate repeated-measures ANOVAs on each Power network revealed main effect of cognitive control in $\operatorname{FPN}(F(2,51)=$ 4.081, $p<0.05)$, Ventral Attention (Vat; $(F(2,51)=5.935, p<$ $0.05)$, and Visual Networks $(F(2,51)=5.306, p<0.05)$. Of these cognitive systems, only the FPN exhibited a control-bytask interaction $(F(2,102)=5.117, p=0.008)$.

\section{Quantifying network reorganization using information theory}

While modularity can be used as an estimate of wholebrain integration or segregation amongst brain modules, it cannot capture module composition or changes in modular partitions. Thus, we used mutual information and variation of information to quantify the similarity of module assignments between high and low cognitive-control conditions in the RiSE encoding, RiSE recognition, and DPX tasks, which we interpret as measures of network reorganization.

Separate repeated-measures ANOVAs examining mutual information and variation of information scores comparing high and low cognitive-control condition partitions in the RiSE encoding, RiSE recognition, and DPX tasks identified a significant effect of task $(F(2,51)=94.558, p<0.001, F(2,51)=$ $78.11, p<0.001$ respectively; Fig. 4), indicating that different levels of network reorganization were observed across tasks. Post hoc t-tests of mutual information scores identified significant differences between each task: DPX $>\operatorname{RiSE}_{\text {Recognition }}(t=$ $5.726, p<0.001), \mathrm{DPX}>\operatorname{RiSE}_{\text {Encoding }}(t=12.436, p<0.001)$, $\operatorname{RiSE}_{\text {Recognition }}>\operatorname{RiSE}_{\text {Encoding }}(t=8.673, p<0.001)$. Post hoc ttests of variation of information scores revealed significant

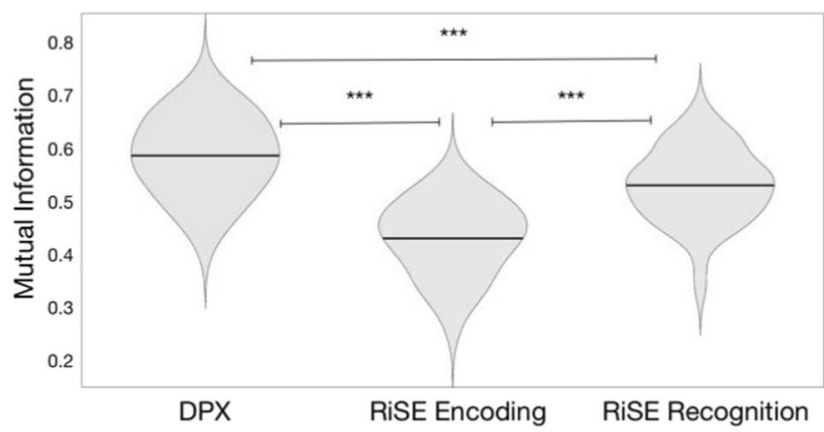

Fig. 4 Mutual information (left) and Variation of Information (right) scores quantifying the similarity of module partitions between high and low cognitive control conditions in the DPX, RiSE encoding, and RiSE differences between each task in the opposite direction as mutual information scores: DPX $<\operatorname{RiSE}_{\text {Recognition }}(t=7.469, p<$ $0.001), \mathrm{DPX}<\operatorname{RiSE}_{\text {Encoding }}(t=16.110, p<0.001)$, $\operatorname{RiSE}_{\text {Recognition }}<\operatorname{RiSE}_{\text {Encoding }}(t=10.799, p<0.001)$. It can be seen in Fig. 4 that the greatest agreement between modular partitions for high and low control conditions (quantified via both Mutual Information and Variation of Information) was observed in the DPX task, followed by RiSE recognition, with the least agreement found for RiSE encoding. Considering that both larger mutual information scores and smaller variation of information scores indicate fewer differences between two modular partitions, we infer that whole-brain reconfiguration of brain networks was lowest between the DPX conditions and greatest during the RiSE encoding conditions.

Subsequent examination of network-specific Variation of Information scores of cognitive systems exhibiting withinnetwork control effects (FPN, Vat, Visual Networks) was performed. A repeated-measures ANOVA identified a main effect of task $(F(2,102)=49.537, p<0.001)$ and main effect of network $(F(2,102)=19.965, p<0.001)$, indicating that the extent of network reorganization differed across tasks and networks. Post hoc t-tests revealed that Variation of Information was greater in the FPN and VAt compared to the Visual Network $(t=5.897, p<0.001$ and $t=5.254, p<0.001$, respectively), but there was no difference between the FPN and VAt.

\section{Participation coefficient}

We used the participation coefficient to assess changes in the diversity of intermodular connections within the FPN associated with cognitive control. We observed a main effect of cognitive control across the three tasks examined where PC was greater during high cognitive-control conditions compared to low cognitive-control conditions $(F(2,51)=4.083$, $p=0.046$; Fig. 5). This finding indicates that the FPN exhibits greater between-module communication (i.e., integration) during high cognitive-control conditions relative to low cognitive-control conditions.

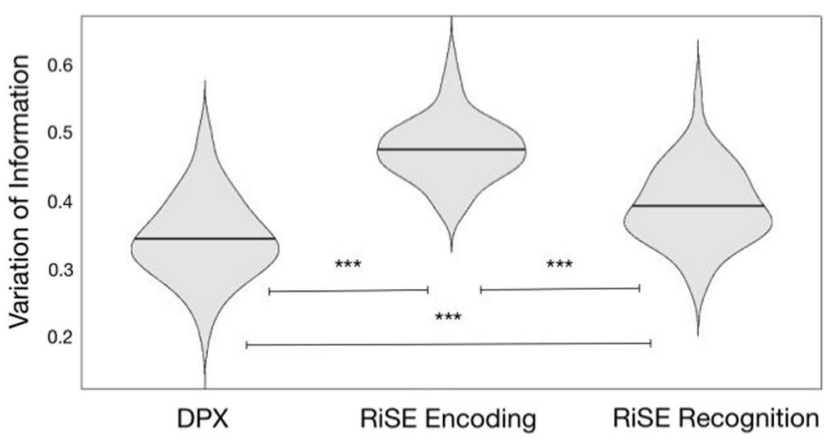

recognition tasks. The horizontal line inside each violin indicates the mean. $* * *$ indicates $\mathrm{p}<0.001$ 


\section{Participation Coefficient Across Tasks}

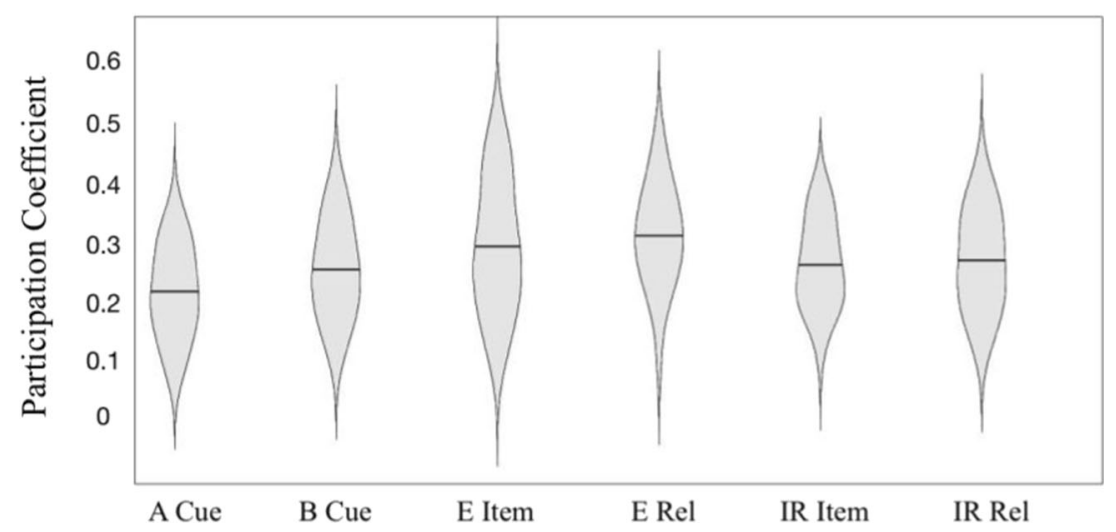

Fig. 5 Participation coefficient (PC) of the frontal parietal network (FPN) across tasks. PC of FPN nodes was greater during high cognitive-control conditions (e.g., B Cue, E Relation, IR Relation) than low cognitivecontrol conditions (e.g., A Cue, E Item, IR Item; $p=0.046$ ). A Cue and

\section{Interpretations of module composition}

Varying levels of integration and segregation between cognitive systems were observed across the tasks examined. In addition to quantitative modularity, mutual information, and PC differences observed, qualitative visual inspection of grouplevel brain module composition across tasks (Fig. 6) revealed that nodes associated with low-level perceptual functioning were consistently constrained to two similar modules across tasks. This included nodes associated with sensorimotor (hand - royal blue, mouth - navy), auditory (orange), and cerebellar networks (yellow). Medial and lateral visual nodes (red) integrated into a single module during the DPX task but segregated into separate modules during the RiSE task with medial nodes integrating with memory nodes (cyan) during encoding and DMN nodes during recognitions conditions (Fig. 6). Nodes associated with high-level cognitive functioning displayed complex levels of module reorganization across tasks. Notably, the FPN (spring green) displayed varying levels of integration and segregation across tasks as it integrated with elements of default mode and salience (sky blue) networks in multiple modules across the RiSE conditions but segregated into the main element of a single module in both DPX conditions.

\section{Discussion}

The purpose of the current study was to quantify and characterize changes in whole-brain network dynamics as they pertain to cognitive control. While previous functional connectivity studies have largely focused on changes within a single cognitive system, or between a set of systems (Dosenbach et al., 2008; Fornito et al., 2012; Hearne et al., 2015), the present study explored context-dependent integration and
B Cue refer to the two DPX conditions. The horizontal line in each violin indicates the mean. E Item and E Rel refer to the RiSE item and relational encoding conditions, respectively. IR Item and IR Rel refer to the RiSE item and relational Recognition conditions, respectively

segregation of brain-wide systems in healthy adults performing fMRI tasks from two distinct cognitive domains that varied in demands for cognitive control as well as demands for episodic mnemonic functions. In doing so, we leverage opposing community structure in the RiSE and DPX tasks to highlight the dynamic reorganizations that support these differentially engaging cognitive control tasks.

\section{Task based modularity}

Examining modularity of the RiSE and DPX tasks revealed different levels of network integration and segregation. Across all tasks, modularity was lower in high control relative to low control conditions. Decreased modularity indicates a reduced proportion of within-module connections in a network, and thus a greater proportion of between-module connections, suggesting a greater level of brain-wide community integration. Similar findings of increased integration have been reported in a range of executive functions including inhibition (Spielberg et al., 2015) and deductive reasoning (Cocchi et al., 2013a; Hearne et al., 2017), and in episodic memory where lower whole-brain modularity has been associated with improved performance (Cohen \& D'Esposito, 2016; Geib et al., 2017; Westphal et al., 2017). Interestingly, a task-by-control interaction effect was also present, indicating that while modularity was greater in low cognitive-control conditions, the extent of the effect varied across tasks. Interaction effects suggest that modules were more segregated in the DPX task than in the RiSE recognition and encoding tasks. This finding is not surprising considering that the DPX primarily engages cognitive control, whereas the RiSE task requires recruitment of both control and long-term (episodic) memory systems. Cohen and colleagues report similar reduced modularity in a memory task, which is thought to require coordination across multiple brain networks, compared to a motor task that likely 

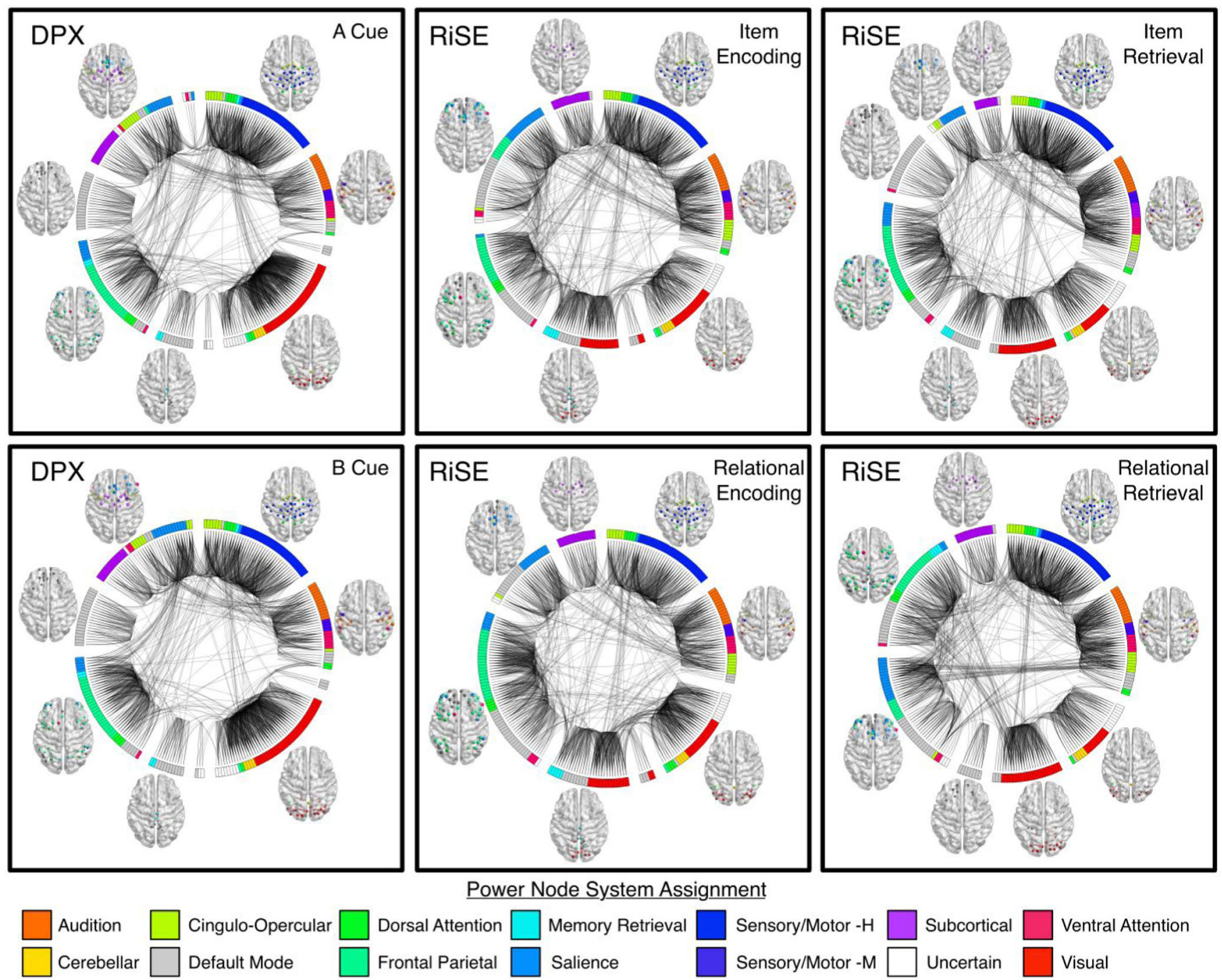

Ventral Attention

Fig. 6 Brain graphs of healthy adults performing the DPX (left column), RiSE encoding (middle column), and RiSE recognition (right column) tasks. Low cognitive-control conditions for each task are shown on the top row, high cognitive control conditions are shown on the bottom row. Edges displayed in each graph represent the strongest $5 \%$ of functional

connections, groups of nodes indicate their module assignment defined using the Louvain Modularity algorithm in the BCT. The color of each node corresponds to one of the 14 cognitive systems proposed in Power et al. (2011). Graphs were visualized using BrainNet Viewer and Circos

involves only a single brain network (Cohen \& D'Esposito, 2016).

We also found that the task-based functional partitions provided a significantly greater modularity score than the restingstate Power partition. Most comparisons between task and rest FC have observed high correspondence (Cole et al., 2014; Fair et al., 2007; Fox \& Raichle, 2007; Greicius et al., 2008). However, more recent comparisons between task and rest functional connectivity have emphasized differences in connectivity patterns (Buckner et al., 2013; Hermundstad et al., 2013; Mennes et al., 2012). Although our sample does not include resting-state fMRI, it is clear that the task-based community structure we observed does not fit the task-negative partition proposed by Power et al. (2011). This is further supported by a recent study from Hearne and colleagues showing that increases in reasoning complexity resulted in a merging of

resting state modules (Hearne et al., 2017). One source of this disagreement between rest and task community partitions may be due to differences in methods for community detection (Infomap compared to Louvain modularity), furthermore the beta-series approach used in the current analysis does not regress out task structure. Nevertheless, the disparity between the presented task-based modular partitions and the proposed Power resting-state subgraph partition do provide support for the concept of differences between task and resting functional architectures.

\section{Network reorganization}

Different levels of network reorganization were observed across tasks. We used two information theory measures, mutual information and variation of information, to quantify 
dynamic network reorganization between modular partitions of high and low cognitive-control conditions. We observed the highest mutual information and lowest VI (i.e., agreement in module composition) in the DPX task, followed by RiSE recognition, and the least mutual information and highest VI during the RiSE encoding. These relationships across tasks were present when examining network-specific VI in the FPN, VAt, and visual systems; however VI in visual nodes was lower than FPN and VAt across all tasks This suggests that changes in connectivity within FPN and VAt likely contribute more to network reorganization between high and low cognitive-control systems than visual nodes. While there was no significant correlation between brain-wide mutual information (I) between high and low CC conditions and modularity scores (Q) for any of the task conditions examined, similar effects across tasks were present. In other words, the DPX was most modular (Q) and contained the greatest mutual information whereas RiSE encoding was least modular $(\mathrm{Q})$ and contained the least amount of mutual information. Incorporating the current findings with previous studies investigating FPN-specific functional connectivity changes associated with cognitive control on the same sample (Ray et al., 2017), the DPX task displayed the greatest change in withinFPN functional connectivity between high and low CC conditions (Ray et al., 2017), and our current work suggests it is also most modular (i.e., greatest network segregation) and experiences the least amount of network reorganization. Conversely, the RiSE-encoding task displayed the least within FPN change in function connectivity between high and low CC conditions (Ray et al., 2017), while current work shows RiSE encoding to be least modular yet undergoes the most network reorganization. All the while, performance on these tasks is significantly correlated, suggesting that they share common processes associated with cognitive control (Sheffield et al., 2014) and they elicit increased activity in the dorsolateral prefrontal and parietal cortices in fMRI studies (Lopez-Garcia et al., 2015; Ragland et al., 2015). Together, this may suggest that cognitive-control processing may be recruited via mechanisms that enhance within network and between network functional connectivity.

\section{Interpretations of module composition}

The FPN exhibited greater integrative properties during high $\mathrm{CC}$ control conditions than low $\mathrm{CC}$ as measured by the participation coefficient. Subsequent qualitative visual inspection of group partitions was performed to better understand changes in module compositions corresponding to changes in modularity $(\mathrm{Q})$, information theory measures (mutual and variation of information), and participation coefficient (PC) across the DPX and RiSE tasks.

Across all conditions examined, several modules were consistently identified that included nodes associated with low- level perceptual systems (e.g., sensorimotor, cerebellar, audition, vision). Nodes in the FPN, DMN, and salience network varied in their module assignments. Focusing on systems exhibiting within-network connectivity effects of cognitive control, the VAt was primarily contained within a single module across all conditions examined, whereas the Visual network typically straddled one or two posterior modules. Conversely, qualitative visual inspection of modules containing FPN nodes were variable across tasks at the group level, where elements the FPN coupled with were either anterior and/or posterior DMN, as well as medial and/or lateral salience systems. Similar findings have been reported in a whole-brain, task-based graph theory analysis comparing modular organizations between 2-back and 0-back conditions in a working memory, executive function n-back task (Braun et al., 2015). Braun and colleagues found that "flexibility," a term defined as the tendency for nodes to change module allegiance, was highest in PFC-related systems, which corresponded with PFC module reorganization, observed in the DPX and RiSE tasks. In relation to episodic memory, Westphal and colleagues report an inverse relationship between DMN and FPN coupling (i.e., network integration) and whole-brain modularity during an episodic memory task (Westphal et al., 2017). This corresponds well with the RiSE task community structure where modules with FPN nodes also contain more DMN nodes and exhibit lower modularity relative to DPX community structure.

The notion of context-dependent dynamic integration and segregation of higher cognitive systems has recently been introduced by other investigative groups. For example Menon and colleagues have suggested that the activity of the frontal parietal and cingulo-opercular systems is related to the behavior of the DMN (Bressler \& Menon, 2010; Menon \& Uddin, 2010). The DMN is commonly referred to as "task negative" because its activity decreases during external goal-oriented actions and increases during performance of tasks requiring self-related processing (Raichle \& Snyder, 2007). Several early studies have supported the hypothesis of a functional antagonism between the two task-positive FPN and cinguloopercular systems on the one hand and the task-negative DMN on the other (Kelly et al., 2008). Decreased activity within the DMN is inversely correlated with cognitive control (Lawrence et al., 2003; McKiernan et al., 2003) and positively correlated with task-unrelated mental activity (Mason et al., 2007), suggesting that they would be segregated systems. Recent advances in graph theory analysis have led to findings that challenge the notion that functional segregation between regions within default-mode and control networks invariably support cognitive task performance (Cocchi et al., 2013b; Fornito et al., 2012; Hearne et al., 2015). Fornito et al. (2012) identified a division of the DMN into core and transitional subsystems where the latter facilitates integration between the core DMN and FPN during goal-directed 
recollection. Moreover it has been shown that increased cognitive demand during cognitive reasoning is accompanied by a loss of segregation and a progressive enhancement of connectivity between control and default-mode networks (Hearne et al., 2015). Related to this, Hearne et al., (2017) also found that increases in reasoning complexity were associated with greater connectivity and more variable community assignment of the FPN. While these previous studies have focused on direct relationships between select cognitive systems, our whole-brain findings complement this more recent view of a context-dependent coordination of task-positive and tasknegative brain systems to support healthy cognitive function. With all of this in consideration, there is compelling evidence suggesting that cognitive control is implemented through the flexible reconfiguration of the FPN as it pertains to a wide range of cognitive domains (Cocchi et al., 2013a; Cole et al., 2013; Fornito et al., 2012; Hearne et al., 2017; Ray et al., 2017; Sheffield et al., 2015).

\section{Methodological considerations}

While the current study provides novel insights into the dynamic brain network reorganization that support cognitive control processing, it is important to acknowledge potential methodological limitations. Recent studies have shown that movement during fMRI acquisition causes systematic changes in functional connectivity (Power et al., 2014; Power et al., 2015; Satterthwaite et al., 2012). To reduce this potential source of error, we took the precaution of performing a "beta-scrubbing" procedure analogous to scrubbing procedures used in restingstate fMRI studies where beta-images containing frame-wise displacement values greater than $0.5 \mathrm{~mm}$ motion were excluded from beta-series. Notably, preliminary modularity analyses performed on these data prior to our "beta-scrubbing" procedure aimed at eliminating trials with excess motion were highly consistent with modular partitions currently presented.

The utility of the Power atlas has been well supported within the neuroimaging community, as numerous studies have utilized this set of regions of interest for various networkconnectivity analyses. However, it is important to note that the cognitive labels assigned to their networks that have been subsequently adopted in the current study as "cognitive systems" are based upon reverse inference. Furthermore, the modularity approach employed, Louvain Modularity, relies on the assumption that nodes may only be assigned to a single module. While this is common in modularity algorithms applied to brain-imaging data, we recognize the plausibility that multifunctional nodes may be involved in more than one module.

Stringent thresholds applied in the current analysis ensure that only strong, positive connections were examined (Zalesky et al., 2016). This is standard practice in the overwhelming majority of graph theoretical analyses; however, it should be noted that this step might inadvertently discard neurobiologically relevant information. Furthermore, to support the reliability of the presented results, replication of these analyses using a $10 \%$ threshold on functional connectivity graphs was highly consistent. While all modularity values were lower at the $10 \%$ threshold, the pattern of results was consistent where task partitions were significantly greater than those of their null and Power partitions. Mutual Information comparing the similarity of partitions from the high and low cognitive-control trials of each task also showed a similar pattern of results where the DPX and RiSE Recognition tasks showed significantly greater mutual information than the RiSE Encoding task. Finally, the module composition of brain graphs remained highly consistent between the two thresholds examined, yielding a high mean mutual information score (MI $=0.84)$ and low mean variation of information $(\mathrm{VI}=0.1136)$ across the six brain graphs (representing the two trial types from each of the three tasks).

\section{Conclusions}

These findings provide insight into how brain networks reorganize to support cognitive performance under differing task contexts. Results suggest that the FPN can contribute to taskappropriate responses through two different mechanisms. Enhanced within-network connectivity in the FPN network is sufficient to support proactive cognitive control, as seen during the DPX. Enhanced network connectivity has also been reported with FPN in the RiSE (Ray et al., 2017); however, the FPN also exhibits the capability to support a wide range of executive functions by flexibly reorganizing into unique community structures that display varying levels of integration and segregation with elements of the DMN, salience, and memory networks to support different forms of encoding and retrieval.

Acknowledgements The authors would like to thank the participants in this study, who gave generously of their time. This research was supported by a research grant from the National Institute of Mental Health (5R01MH059883). All authors approved the final version of the paper for submission.

\section{References}

Badre, D. (2008). Cognitive control, hierarchy, and the rostrocaudal organization of the frontal lobes. Trends in Cognitive Sciences, 12(5), 193-200. https://doi.org/10.1016/j.tics.2008. 02.004

Banich, M. T. (1997). Neuropsychology: The neural bases of mental function.

Banich, M. T., Milham, M. P. P., Atchley, R. A., Cohen, N. J., Webb, A., Wszalek, T., et al. (2000). Prefrontal regions play a predominant role in imposing an attentional "set": evidence 
from fMRI. Brain Research. Cognitive Brain Research, 10(12), 1-9.

Bassett, D. S., Wymbs, N. F., Porter, M. A., Mucha, P. J., Carlson, J. M., \& Grafton, S. T. (2011). Dynamic reconfiguration of human brain networks during learning. Proceedings of the National Academy of Sciences, 108(18), 7641-7646. https://doi.org/10.1073/pnas.1018985108

Braun, U., Schäfer, A., Walter, H., Erk, S., Romanczuk-Seiferth, N., Haddad, L., et al. (2015). Dynamic reconfiguration of frontal brain networks during executive cognition in humans. Proceedings of the National Academy of Sciences, 112(37), 11678-11683. https://doi.org/10.1073/pnas. 1422487112

Bressler, S. L., \& Menon, V. (2010). Large-scale brain networks in cognition: emerging methods and principles. Trends in Cognitive Sciences, 14(6), 277-290. https://doi.org/10.1016/j.tics.2010.04. 004

Buckner, R. L. L., Krienen, F. M., \& Yeo, B. T. T. (2013). Opportunities and limitations of intrinsic functional connectivity MRI. Nature Publishing Group, 16(7), 832-837. https://doi.org/10.1038/nn.3423

Bullmore, E. T., \& Bassett, D. S. (2011). Brain Graphs: Graphical Models of the Human Brain Connectome. Annual Review of Clinical Psychology, 7(1), 113-140. https://doi.org/10.1146/annurevclinpsy-040510-143934

Cocchi, L., Halford, G. S., Zalesky, A., Harding, I. H., Ramm, B. J., Cutmore, T., et al. (2013a). Complexity in Relational Processing Predicts Changes in Functional Brain Network Dynamics. Cerebral Cortex, 24(9), 2283-2296. https://doi.org/10.1093/cercor/ bht075

Cocchi, L., Zalesky, A., Fornito, A., \& Mattingley, J. B. (2013b). Dynamic cooperation and competition between brain systems during cognitive control. Trends in Cognitive Sciences, 17(10), 493501. https://doi.org/10.1016/j.tics.2013.08.006

Cohen, J. R., \& D'Esposito, M. (2016). The Segregation and Integration of Distinct Brain Networks and Their Relationship to Cognition. Journal of Neuroscience, 36(48), 12083-12094. https://doi.org/10. 1523/JNEUROSCI.2965-15.2016

Cole, M. W., \& Schneider, W. (2007). The cognitive control network: Integrated cortical regions with dissociable functions. NeuroImage, 37(1), 343-360. https://doi.org/10.1016/j.neuroimage.2007.03.071

Cole, M. W., Bassett, D. S., Power, J. D., Braver, T. S., \& Petersen, S. E. E. (2014). Intrinsic and Task-Evoked Network Architectures of the Human Brain. Neuron, 83(1), 238-251. https://doi.org/10.1016/j. neuron.2014.05.014

Cole, M. W., Reynolds, J. R., Power, J. D., Repovs, G., Anticevic, A., \& Braver, T. S. (2013). Multi-task connectivity reveals flexible hubs for adaptive task control. Nature Publishing Group, 16(9), 13481355. https://doi.org/10.1038/nn.3470

Crossley, N. A., Mechelli, A., Vértes, P. E., Winton-Brown, T. T., Patel, A. X., Ginestet, C. E., et al. (2013). Cognitive relevance of the community structure of the human brain functional coactivation network. Proceedings of the National Academy of Sciences, 110(28), 11583-11588. https://doi.org/10.1073/pnas.1220826110

Dosenbach, N. U. F., Fair, D. A., Cohen, A. L., Schlaggar, B. L. L., \& Petersen, S. E. E. (2008). A dual-networks architecture of top-down control. Trends in Cognitive Sciences, 12(3), 99-105. https://doi.org/ 10.1016/j.tics.2008.01.001

Fair, D. A., Dosenbach, N. U. F., Church, J. A., Cohen, A. L., Brahmbhatt, S., Miezin, F. M., et al. (2007). Development of distinct control networks through segregation and integration. Proceedings of the National Academy of Sciences of the United States of America, 104(33), 13507-13512. https://doi.org/10.1073/pnas. 0705843104

Fornito, A., Harrison, B. J., Zalesky, A., \& Simons, J. S. (2012). Competitive and cooperative dynamics of large-scale brain functional networks supporting recollection. Proceedings of the National
Academy of Sciences, 109(31), 12788-12793. https://doi.org/10. 1073/pnas.1204185109

Fox, M. D., \& Raichle, M. E. (2007). Spontaneous fluctuations in brain activity observed with functional magnetic resonance imaging. Nature Reviews Neuroscience, 8(9), 700-711. https://doi.org/10.1038/nrn2201

Geib, B. R., Stanley, M. L., Dennis, N. A., Woldorff, M. G., \& Cabeza, R. (2017). From hippocampus to whole-brain: The role of integrative processing in episodic memory retrieval. Human Brain Mapping, 38(4), 2242-2259. https://doi.org/10. 1002/hbm. 23518

Gratton, C., Laumann, T. O., Gordon, E. M., Adeyemo, B., \& Petersen, S. E. E. (2016). Evidence for Two Independent Factors that Modify Brain Networks to Meet Task Goals. Cell Reports, 17(5), 1276-1288. https://doi.org/10.1016/j. celrep.2016.10.002

Greicius, M. D., Supekar, K., Menon, V., \& Dougherty, R. F. (2008). Resting-State Functional Connectivity Reflects Structural Connectivity in the Default Mode Network. Cerebral Cortex, 19(1), 72-78. https://doi.org/10.1093/ cercor/bhn059

Guimerà, R., \& Nunes Amaral, L. A. (2005). Functional cartography of complex metabolic networks. Nature, 433(7028), 895-900. https:// doi.org/10.1038/nature03288

Hearne, L. J., Cocchi, L., Zalesky, A., \& Mattingley, J. B. (2017). Reconfiguration of brain network architectures between resting state and complexity-dependent cognitive reasoning. The Journal of Neuroscience, 0485-17. https://doi.org/10.1523/ JNEUROSCI.0485-17.2017

Hearne, L., Cocchi, L., Zalesky, A., \& Mattingley, J. B. (2015). Interactions between default mode and control networks as a function of increasing cognitive reasoning complexity. Human Brain Mapping, 36(7), 2719-2731. https://doi.org/ 10.1002/hbm.22802

Henderson, D., Poppe, A. B., Barch, D. M., Carter, C. S., Gold, J. M., Ragland, J. D., et al. (2012). Optimization of a Goal Maintenance Task for Use in Clinical Applications. Schizophrenia Bulletin, 38(1), 104-113. https://doi.org/10. 1093/schbul/sbr172

Hermundstad, A. M., Bassett, D. S., Brown, K. S., Aminoff, E. M., Clewett, D., Freeman, S., et al. (2013). Structural foundations of resting-state and task-based functional connectivity in the human brain. Proceedings of the National Academy of Sciences, 110(15), 6169-6174. https://doi.org/10.1073/pnas. 1219562110

Kelly, A. M. C., Uddin, L. Q., Biswal, B. B. B., Castellanos, F. X. X., \& Milham, M. P. P. (2008). Competition between functional brain networks mediates behavioral variability. Connectivity Connectivity, 39(1), 527-537. https://doi.org/10.1016/j. neuroimage.2007.08.008

Lawrence, N. S., Ross, T. J., Hoffmann, R., Garavan, H., \& Stein, E. A. (2003). Multiple Neuronal Networks Mediate Sustained Attention. Journal of Cognitive Neuroscience, 15(7), 10281038. https://doi.org/10.1162/089892903770007416

Lopez-Garcia, P., Lesh, T. A., Salo, T., Barch, D. M., MacDonald, A. W., III, Gold, J. M., et al. (2015). The neural circuitry supporting goal maintenance during cognitive control: a comparison of expectancy AX-CPT and dot probe expectancy paradigms. Cognitive, Affective, \& Behavioral Neuroscience. https://doi.org/10.3758/s13415-015-0384-1

MacDonald, A. W. (2000). Dissociating the Role of the Dorsolateral Prefrontal and Anterior Cingulate Cortex in Cognitive Control. Science, 288(5472), 1835-1838. https:// doi.org/10.1126/science.288.5472.1835

Mason, M. F., Norton, M. I., Van Horn, J. D., Wegner, D. M., Grafton, S. T., \& Macrae, C. N. (2007). Wandering Minds: 
The Default Network and Stimulus-Independent Thought. Science, 315(5810), 393-395. https://doi.org/10.1126/science. 1131295

McKiernan, K. A., Kaufman, J. N., Kucera-Thompson, J., \& Binder, J. R. (2003). A Parametric Manipulation of Factors Affecting Taskinduced Deactivation in Functional Neuroimaging. Journal of Cognitive Neuroscience, 15(3), 394-408. https://doi.org/10.1162/ 089892903321593117

Meilă, M. (2007). Comparing clusterings - an information based distance. Journal of Multivariate Analysis, 98(5), 873-895. https://doi.org/10.1016/j.jmva.2006.11.013

Mennes, M., Kelly, C., Colcombe, S., Castellanos, F. X. X., \& Milham, M. P. P. (2012). The Extrinsic and Intrinsic Functional Architectures of the Human Brain Are Not Equivalent. Cerebral Cortex (New York, N.Y. : 1991), 23(1), 223-229. https://doi.org/10.1093/cercor/bhs010

Menon, V., \& Uddin, L. Q. (2010). Saliency, switching, attention and control: a network model of insula function. Brain Structure and Function, 214(5-6), 655-667. https://doi.org/ 10.1007/s00429-010-0262-0

Meunier, D. (2009). Hierarchical modularity in human brain functional networks. Frontiers in Neuroinformatics, 3. https://doi. org/10.3389/neuro.11.037.2009

Mumford, J. A., Turner, B. O., Ashby, F. G., \& Poldrack, R. A. (2012). Deconvolving BOLD activation in event-related designs for multivoxel pattern classification analyses. NeuroImage, 59(3), 2636-2643. https://doi.org/10.1016/j. neuroimage.2011.08.076

Murray, L. J., \& Ranganath, C. (2007). The Dorsolateral Prefrontal Cortex Contributes to Successful Relational Memory Encoding. Journal of Neuroscience, 27(20), 55155522. https://doi.org/10.1523/JNEUROSCI.0406-07.2007

Newman, M. E. J. (2004). Fast algorithm for detecting community structure in networks. Physical Review E, 69(6), 066133. https://doi.org/10.1103/PhysRevE.69.066133

Niendam, T. A., Laird, A. R., Ray, K. L., Dean, Y. M., Glahn, D. C., \& Carter, C. S. (2012). Meta-analytic evidence for a superordinate cognitive control network subserving diverse executive functions. Cognitive, Affective, \& Behavioral Neuroscience, 12(2), 241-268. https://doi.org/10.3758/ s13415-011-0083-5

Poppe, A. B., Barch, D. M., Carter, C. S., Gold, J. M., Ragland, J. D., Silverstein, S. M., \& MacDonald, A. W., III. (2016). Reduced Frontoparietal Activity in Schizophrenia Is Linked to a Specific Deficit in Goal Maintenance: A Multisite Functional Imaging Study. Schizophrenia Bulletin, 42(5), sbw036-1157. https://doi.org/10.1093/schbul/sbw036

Power, J. D., Cohen, A. L., Nelson, S. M., Wig, G. S., Barnes, K. A., Church, J. A., et al. (2011). Functional Network Organization of the Human Brain. Neuron, 72(4), 665-678. https://doi.org/10.1016/j.neuron.2011.09.006

Power, J. D., Mitra, A., Laumann, T. O., Snyder, A. Z., Schlaggar, B. L. L., \& Petersen, S. E. E. (2014). Methods to detect, characterize, and remove motion artifact in resting state fMRI. NeuroImage, 84,320 341. https://doi.org/10.1016/j.neuroimage.2013.08.048

Power, J. D., Schlaggar, B. L. L., \& Petersen, S. E. E. (2015). Recent progress and outstanding issues in motion correction in resting state fMRI. NeuroImage, 105, 536-551. https://doi. org/10.1016/j.neuroimage.2014.10.044

Ragland, J. D., Blumenfeld, R. S., Ramsay, I. S., Yonelinas, A. P., Yoon, J. H., Solomon, M., et al. (2012). Neural correlates of relational and item-specific encoding during working and long-term memory in schizophrenia. NeuroImage, 59(2), 1719-1726. https://doi.org/10.1016/j.neuroimage.2011.08.055

Ragland, J. D., Ranganath, C., Harms, M. P., Barch, D. M., Gold, J. M., Layher, E., et al. (2015). Functional and
Neuroanatomic Specificity of Episodic Memory Dysfunction in Schizophrenia: A Functional Magnetic Resonance Imaging Study of the Relational and Item-Specific Encoding Task. JAMA Psychiatry, 72(9), 909-916. https://doi.org/10.1001/ jamapsychiatry.2015.0276

Ragland, J., Laird, A. R., Ranganath, C., Blumenfeld, R., Gonzales, S. M., \& Glahn, D. C. (2009). Prefrontal Activation Deficits During Episodic Memory in Schizophrenia. American Journal of Psychiatry, 166(8), 863-874. https://doi.org/10.1176/appi.ajp. 2009.08091307

Raichle, M. E., \& Snyder, A. Z. (2007). A default mode of brain function: a brief history of an evolving idea. NeuroImage, 37(4), 1083-90- discussion 1097-9. https://doi.org/10.1016/j.neuroimage.2007.02.041

Ray, K. L., Lesh, T. A., Howell, A. M., Salo, T. P., Ragland, J. D., MacDonald, A. W., et al. (2017). Functional network changes and cognitive control in schizophrenia. NeuroImage. Clinical, 15, 161170. https://doi.org/10.1016/j.nicl.2017.05.001

Rubinov, M., \& Sporns, O. (2010). Complex network measures of brain connectivity: Uses and interpretations. NeuroImage, 52(3), 1059-1069. https://doi.org/10.1016/j.neuroimage.2009. 10.003

Rubinov, M., \& Sporns, O. (2011). Weight-conserving characterization of complex functional brain networks. Connectivity Connectivity, 56(4), 2068-2079. https://doi.org/10.1016/j. neuroimage.2011.03.069

Satterthwaite, T. D., Wolf, D. H., Loughead, J., \& Ruparel, K. (2012). Impact of in-scanner head motion on multiple measures of functional connectivity: Relevance for studies of neurodevelopment in youth. NeuroImage.

Sheffield, J. M., Gold, J. M., Strauss, M. E., Carter, C. S., MacDonald, A. W., III, Ragland, J. D., et al. (2014). Common and specific cognitive deficits in schizophrenia: relationships to function. Cognitive, Affective, \& Behavioral Neuroscience, 14(1), 161-174. https://doi.org/10.3758/ s13415-013-0211-5

Sheffield, J. M., Repovs, G., Harms, M. P., Carter, C. S., Gold, J. M., MacDonald, A. W., III, et al. (2015). Fronto-parietal and cingulo-opercular network integrity and cognition in health and schizophrenia. Neuropsychologia, 73 IS, 82-93.

Spielberg, J. M., Miller, G. A., Heller, W., \& Banich, M. T. (2015). Flexible brain network reconfiguration supporting inhibitory control. Proceedings of the National Academy of Sciences, 112(32), 10020-10025.

Sporns, O. (2018). Graph theory methods: applications in brain networks. Dialogues in Clinical Neuroscience, 20(2), 111121. https://doi.org/10.1016/j.neuroimage

Sporns, O., \& Betzel, R. F. (2016). Modular Brain Networks. Annual Review of Psychology, 67, 613-640. https://doi.org/ 10.1146/annurev-psych-122414-033634

Sporns, O., Honey, C. J., \& Kotter, R. (2007). Identification and classification of hubs in brain networks. PLOS ONE, 2(10), e1049-14. https://doi.org/10.1371/journal.pone.0001049

Turner, B. O., Mumford, J. A., Poldrack, R. A., \& Ashby, F. G. (2012). Spatiotemporal activity estimation for multivoxel pattern analysis with rapid event-related designs. NeuroImage, 62(3), 1429-1438. https://doi.org/10.1016/j.neuroimage.2012. 05.057

Veen, V. V., \& Carter, C. S. (2006). Conflict and Cognitive Control in the Brain. Current Directions in Psychological Science, 15(5), 237-240. https://doi.org/10.1111/j.1467-8721. 2006.00443.x

Westphal, A. J., Wang, S., \& Rissman, J. (2017). Episodic Memory Retrieval Benefits from a Less Modular Brain Network Organization. The Journal of Neuroscience, 37(13), 3523-3531. https://doi.org/10.1523/JNEUROSCI.2509-16. 2017 
Zalesky, A., Fornito A., Bullmore, E. T. (2010). "Network-based statistic: identifying differences in brain networks." Neuroimage, 53.4, 1197-1207.

Zalesky, A., Fornito, A., Cocchi, L., Gollo, L. L., van den Heuvel, M. P., \& Breakspear, M. (2016). Connectome sensitivity or specificity: which is more important? NeuroImage, 142, 407-420. https://doi. org/10.1016/j.neuroimage.2016.06.035
Publisher's note Springer Nature remains neutral with regard to jurisdictional claims in published maps and institutional affiliations.

None of the data or materials for the experiments reported here are available, and none of the experiments were preregistered. 